TRANSACTIONS OF THE

AMERICAN MATHEMATICAL SOCIETY

Volume 334, Number 1, November 1992

\title{
CHARACTERISTIC CYCLES OF HOLOMORPHIC DISCRETE SERIES
}

\author{
JEN-TSEH CHANG
}

\begin{abstract}
The characteristic cycles of standard modules arising from closed $K$-orbits in a flag variety are studied and those of holomorphic discrete series are determined explicitly. Also the asymptotic expansion of the distribution characters of holomorphic discrete series are computed; the result verifies a conjecture of D. Vogan in this special case.
\end{abstract}

Suppose $G_{\mathbb{R}}$ is a linear semisimple Lie group and $K_{\mathbb{R}}$ a maximal compact subgroup of $G_{\mathbb{R}}$. In case the symmetric space $G_{\mathbb{R}} / K_{\mathbb{R}}$ carries a Hermitian structure, $G_{\mathbb{R}}$ has a family of holomorphic discrete series representations. Let $I$ be the underlying Harish-Chandra module of an arbitrary holomorphic discrete series representation and $\Theta$ be the corresponding distribution character on the group. The associated cycle $\mathscr{V}(I)$ of $I$ is an algebraic cycle defined algebraically on $\mathfrak{g}^{*}$, the dual to the complexification of the Lie algebra $\mathfrak{g}_{\mathbb{R}}$ of $G_{\mathbb{R}}$. On the other hand, according to [Barbasch-Vogan], the Fourier transform of the leading term of the asymptotics of $\Theta$ near the identity is a linear combination of canonical invariant measures on coadjoint $G_{\mathbb{R}}$-orbits in $\mathfrak{g}_{\mathbb{R}}^{*}$. It therefore determines a cycle on $\mathfrak{g}_{\mathbb{R}}^{*}$. In this paper we determine both invariants attached to the holomorphic discrete series. It then verifies in this special case a conjecture of D. Vogan which asserts that the "major" part of both invariants coincide under the orbit correspondence of [Sekiguchi].

The complexified tangent space of $G_{\mathbb{R}} / K_{\mathbb{R}}$ at the identity coset is naturally identified to a quotient of $\mathfrak{g}$, therefore, via the invariant nondegenerate Killing form, the holomorphic tangent space is identified to a subspace in $\mathfrak{g}^{*}$. Here the holomorphic structure is the one chosen to define $I$. Then the associated cycle $\mathscr{V}(I)$ is the holomorphic tangent space with multiplicity the dimension of the lowest $K_{\mathbb{R}}$-type of $I$ (2.13). The computation is made through the corresponding $\mathscr{D}$-module on the flat variety $X$ of $\mathfrak{g}$ and the moment map from the cotangent bundle $T^{*} X$ to $\mathfrak{g}^{*}$. Generally $\mathscr{D}$-modules giving rise to the discrete series are supported on closed $K$-orbits on $X$ ( $K$ is the complexification of $K_{\mathbb{R}}$ ), the normal derivatives then induce a good filtration on these $\mathscr{D}$-modules. This then computes the associated graded modules as sheaves of coherent $\mathscr{O}$ modules on $T^{*} X$. Although in general the moment map is quite complicated on the support of the associated graded module, in the situation where holo-

Received by the editors September 24, 1990.

1980 Mathematics Subject Classification (1985 Revision). Primary 22E46; Secondary 14F05, $20 \mathrm{G} 20$.

Supported in part by NSF grant DMS-8902352. 
morphic discrete series do arise it becomes quite simple. In fact our results also cover the case where the standard data are Borel-de Siebenthal (Proposition 2.15).

The computation of the asymptotics of $\Theta$ takes on a rather different path. According to Rossmann's Kirillov formula [Rossmann 1], near the origin of $\mathfrak{g}_{\mathbb{R}}$, infinitesimally $\Theta$ is given by the Fourier transform of the canonical invariant measure on the coadjoint $G_{\mathbb{R}}$-orbit (in $\mathfrak{g}_{\mathbb{R}}^{*}$ ) of the infinitesimal character of $I$. Since invariant orbital measures are homogeneous, their asymptotics at the origin is the same as that of orbital measures with parameters (i.e., infinitesimal characters) tending to 0 along a certain direction. When the limit of these orbits contains orbits of the same dimension, the limit is relatively easy to compute (as in [Rossmann 2]). But such an ideal situation rarely occurs; even in the case of holomorphic discrete series. Our observation is that it is possible to fiber the coadjoint orbit in question over an orbit with the nice equal dimension property mentioned above; with fibers isomorphic to coadjoint orbits of some compact group. The orbital integral limit in the setting of compact groups is well known by formulae of Weyl and Kirillov. The integration formula, together with limits on the base and fibers, then computes the limit. The result is that the asymptotics cycle is the nilpotent orbit corresponding to the holomorphic tangent space (as identified in $\mathfrak{g}^{*}$ ) with the same multiplicity mentioned above (Theorem 5.5). The orbit correspondence here is via Sekiguchi's construction: very roughly speaking, it establishes a natural bijection between certain classes of $K$-orbits and $G_{\mathbb{R}}$-orbits in the complex nilpotent cone of $\mathfrak{g}^{*}$.

The schemes of the computations, in both cases, outlined above fit in a much more general context. The specific structure information from the Hermitian situation was assumed in order to carry out the computation; especially in determining the support of the leading term of the asymptotics of $\Theta$. Related results were contained in [King] in which the "character polynomials" of holomorphic discrete series were obtained. We remark that, for general discrete series representations, the situation is much more complicated as indicated in [King] and by the complexity of the moment map. As for the organization of the paper, $\S 1$ establishes the basics of associated cycles of Harish-Chandra modules using the $\mathscr{D}$-modules technique. Section 2 computes the associated cycles arising from closed $K$-orbits in a flag variety under a technical assumption. Section 3 recalls the setup around the asymptotic expansion. The fibration is described in $\S 4$, and the asymptotics for holomorphic discrete series is computed in $\S 5$.

We would like to thank W. Schmid and D. Vogan for useful discussions.

Throughout this paper we consider real Lie groups which have finite index in the real points of a connected complex reductive algebraic group (defined over $\mathbb{R}$ ). This choice is more for the convenience of the writing; most of the settings and results hold in a much more general situation. Let $G_{\mathbb{R}}$ be a such real Lie group and $K_{\mathbb{R}}$ be a fixed maximal compact subgroup of $G_{\mathbb{R}}$ with the corresponding Cartan involution $\theta$. As usual the $(-1)$-eigenspace of $\theta$ in $\mathfrak{g}$ is denoted by $\mathfrak{p}$. As notational convention, deleting the subscript $\mathbb{R}$ means taking complexification. We now quickly recall the basic setup for $\mathscr{D}$-modules on the flag variety $X$ of $\mathfrak{g}$; for details, see [Beilinson-Bernstein]. Let $Z$ be a $K$-orbit 
in $X$ and denote by $i$ the inclusion. According to [Matsuki], $Z$ can also be specified by the data $(\mathfrak{b}, \mathfrak{h})$ consisting of a Borel subalgebra $\mathfrak{b}$ and a Cartan subalgebra $\mathfrak{h} \subset \mathfrak{b}$, which at the same time is the complexification of a real $\theta$ stable Cartan subalgebra $\mathfrak{h}_{\mathbb{R}}$. This pair also determines a positive root system $\Phi^{+}$in $\Phi(\mathfrak{g}, \mathfrak{h})$ so that the nilradical $\mathfrak{n}^{-}$of $\mathfrak{b}$ is spanned by negative root vectors. The $(+1)$-eigenspace of $\theta$ in $\mathfrak{h}$, denoted by $\mathfrak{t}$, is then the Lie algebra of the reductive part of the isotropy subgroup $K_{\mathfrak{b}}$ at $\mathfrak{b}$. A set of standard data is, by definition, a $K$-homogeneous connection together with a compatible infinitesimal character. In other words, it consists of a triple $(Z, \tau, \lambda)$ where $\lambda \in \mathfrak{h}^{*}$ and $\tau$ is a character on $K_{\mathfrak{b}}$ whose derivative satisfies $d \tau=\lambda-\rho$ on $\mathfrak{t}$. As usual, $\rho=\rho\left(\Phi^{+}\right)$denotes the half sum of positive roots. This set of data gives rise to the standard coherent $\mathscr{D}_{\lambda}$-module $\mathscr{I}_{\lambda}=i_{+}($ind $\tau)$ on $X$, where ind $\tau$ is the $K$-homogeneous invertible sheaf on $Z$ induced from the character $\tau$. We use $i_{+}$(resp. $i_{*}$ ) for the direct image functor in the category of $\mathscr{D}$-modules (resp. $\mathscr{O}$-modules). Write $I_{\lambda}$ for its global section space. The subscript will often be omitted if no confusion could occur. Following [BorhoBrylinski], the characteristic cycle of $\mathscr{I}$ is given by

$$
\operatorname{Ch}(\mathscr{I}):=\operatorname{Supp}(\operatorname{gr} \mathscr{I}),
$$

the support with multiplicity of the graded module $\operatorname{gr} \mathscr{I}$ on $T^{*} X$ with respect to a good filtration. Similarly the associated cycle on $\mathfrak{g}^{*}$ of the Harish-Chandra module $I$ is given by

$$
\mathscr{V}(I):=\operatorname{Supp}(\operatorname{gr} I)
$$

The moment map is denoted by $\gamma: T^{*} X \rightarrow \mathfrak{g}^{*}$, given by, over $x \in X$, the natural inclusion $T_{x}^{*} X=\left(\mathfrak{g} / \mathfrak{b}_{x}\right)^{*} \hookrightarrow g^{*}$. Recall that $\mathscr{I}$ has a natural filtration, for $j$ an integer,

1.1. $\mathscr{I}_{j}=$ subsheaf of local sections of $\mathscr{I}$ with degree of transversal derivative $\leq j$.

1.2. Lemma. If $Z$ is a closed $K$-orbit in $X$, then $\left(\mathscr{J}_{j}\right)$ is a good filtration and

$$
\mathscr{I}_{0}=i_{*}\left(\text { ind } \tau \otimes \operatorname{det} \mathscr{N}_{Z \mid X}\right) ; \quad \operatorname{gr}_{j} \mathcal{I}=i_{*}\left(\text { ind } \tau \otimes \operatorname{det} \mathscr{N}_{Z \mid X} \otimes \mathscr{N}_{Z \mid X}^{(j)}\right) .
$$

Here $\mathscr{N}_{Z \mid X}$ denotes the normal sheaf of $Z$ in $X$ and $\mathscr{A}^{(j)}$ stands for the $j$ th symmetric power of $\mathscr{A}$.

Since $Z$ is closed, $i$ is proper and the lemma is immediate. Also note that $Z$ being closed is equivalent to

\section{3. $\Phi^{+}$is $\theta$-stable and contains no real roots.}

Under this assumption, $\mathfrak{t}$ is a Cartan subalgebra of $\mathfrak{k}$ and the (nonzero) restriction of roots in $\Phi^{+}(\mathfrak{g}, \mathfrak{h})$ to $\mathfrak{t}$ gives rise to a positive root system $\Phi^{+}(\mathfrak{k}, \mathfrak{t})$ for the pair $(\mathfrak{k}, \mathfrak{t})$; whose half sum will be denoted by $\rho_{\mathfrak{k}}$. With respect to $\Phi^{+}(\mathfrak{g}, \mathfrak{h})$, a linear functional $\lambda$ on $\mathfrak{h}$ is called dominant if $\langle\lambda, \breve{\alpha}\rangle \notin \mathbb{Z}_{<0}$, for all $\alpha \in \Phi^{+} ; \mathbb{R}$-dominant if $\langle\lambda, \alpha\rangle \geq 0$ for all $\alpha \in \Phi^{+}$. Note that being $\mathbb{R}$-dominant implies being dominant.

1.4. Theorem. Suppose that $Z$ is a closed orbit, $\lambda$ is regular and $\mathbb{R}$-dominant, then $\mathscr{V}(I)=\operatorname{Supp}\left(\gamma_{*} \operatorname{gr} \mathscr{I}\right)$. 
Note that the assumption on $\lambda$ above implies that $I$ is nontrivial. Now Lemma 1.2 says that $\left(\mathscr{F}_{j}\right)$ is a good filtration. In view of $\S 1.6$ of [BorhoBrylinski], the theorem follows from

$$
H^{1}\left(X, \mathscr{I}_{j}\right)=0 \text { if } 0 \ll j .
$$

1.6. Lemma. $H^{k}\left(X, \operatorname{gr}_{j} \mathscr{I}\right)=0$, for all $k>0$ and $j \geq 0$.

We first show that this lemma implies (1.5). From the short exact sequence

$$
0 \rightarrow \mathscr{I}_{j-1} \rightarrow \mathscr{I}_{j} \rightarrow \mathrm{gr}_{j} \mathscr{I} \rightarrow 0
$$

we obtain, by Lemma $1.6, H^{1}\left(X, \mathscr{I}_{j-1}\right) \rightarrow H^{1}\left(X, \mathscr{I}_{j}\right)$, for $j \geq 0$. Now $\mathscr{I}_{j}$ being a coherent $\mathscr{O}_{X}$-module, the above cohomologies are finite-dimensional. Hence the surjections must be stational if $j \geq j_{0}$ for some $j_{0}$. If $H^{1}\left(X, \mathscr{J}_{j_{0}}\right) \neq$ 0 , then $\lim _{\longrightarrow} H^{1}\left(X, \mathscr{J}_{j}\right) \neq 0$, which is just $H^{1}(X, \mathscr{J})$. On the other hand, $\lambda$ being dominant, the cohomology vanishes [Beilinson-Bernstein]. Therefore $H^{1}\left(X, \mathscr{F}_{j}\right)=0$, for $j \geq j_{0}$.

As for Lemma 1.6, note that by Lemma 1.2,

$$
\left.H^{k}\left(X, \operatorname{gr}_{j} \mathscr{I}\right)=H^{k}\left(Z, \text { ind } \tau \otimes \operatorname{det} \mathscr{N}_{Z \mid X} \otimes \mathscr{N}_{Z \mid X}^{(j)}\right)\right)
$$

Since the sheaf $\mathscr{N}_{Z \mid X}$ on $Z$ is induced from the $K \cap B$-module $\mathfrak{n} \cap \mathfrak{p}$ which in turn, when viewed as a subset of the Lie algebra $\mathfrak{g}$, induces global vector fields on $X$. Therefore $\mathscr{N}_{Z \mid X}$ is generated by its global sections on $Z$. According to Theorem $\mathrm{G}$ of [Griffiths], the vanishing statement of 1.6 then follows from

$$
\text { (ind } \left.\tau \otimes \operatorname{det} \mathscr{N}_{Z \mid X}\right)^{*} \otimes \operatorname{det} \mathscr{N}_{Z \mid X} \otimes \mathscr{K}_{Z}<0
$$

on $Z$; here $\mathscr{K}_{Z}$ is the canonical sheaf on $Z$. Now the closed $K$-orbit $Z$ is also the flag variety of the reductive group $K$, and in our setting the negativity condition (1.7) amounts to

$$
\left\langle-\left.d \tau\right|_{\mathfrak{t}}-2 \rho_{\mathfrak{k}}, \alpha\right\rangle<0 \quad \text { for all } \alpha \in \Phi^{+}(\mathfrak{k}, \mathfrak{t}) .
$$

Since $\lambda$ is assumed to be regular and $\mathbb{R}$-dominant w.r.t. $\Phi^{+}(\mathfrak{g}, \mathfrak{h}), d \tau=\lambda-\rho$ is $\mathbb{R}$-dominant w.r.t. the same positive system. $\Phi^{+}(\mathfrak{k}, \mathfrak{t})$ being the restriction of the same system, (1.7) follows. This completes the proof of Theorem 1.4. We remark that in case $\mathfrak{h}=\mathfrak{t}$, the vanishing result above is precisely Lemma 5.2 in [Schmid 1].

We conclude this section with the following remarks. The regularity assumption on $\lambda$ in Theorem 1.4 is in fact not necessary: the conclusion of the theorem remains true if $\lambda$ is $\mathbb{R}$-dominant and $I$ is nontrivial. In fact similar result holds in a much more general setting: when the Harish-Chandra module $I$ is realized on a maximally real $K$-orbit (the top data in the sense of [Chang]) under suitable assumption on $\lambda$. We will not use these results and will not go into the technicality here.

In this section, we assume that

\section{1. $G_{\mathbb{R}}$ contains a compact Cartan subgroup.}

According to [Harish-Chandra], this is equivalent to " $G_{\mathbb{R}}$ has discrete series representations." We now formulate the parameterization of the discrete series 
in terms of the setup in $\S 1$. Note that under the condition 2.1 , the Cartan subalgebra $\mathfrak{h}_{\mathbb{R}}$ used to specify any closed $K$-orbit in the flag variety $X$ is necessarily compact. Denote by $R_{d}\left(G_{\mathbb{R}}\right)$ the set of Harish-Chandra modules of the discrete series of $G_{\mathbb{R}}$.

2.2. Proposition. $R_{d}\left(G_{\mathbb{R}}\right)$ consists of all standard modules associated to data $(Z, \tau, \lambda)$ with $Z$ a closed $K$-orbit, $\lambda$ regular and $\mathbb{R}$-dominant.

This result is well known; for a reference see 9.4 of [Chang]. In case $\lambda$ is $\mathbb{R}$ dominant but not regular, the resulting standard module (if nontrivial) is then one of the so-called limits of discrete series. We now fix one set of such data, i.e., $(Z, \tau, \lambda)$ with

\section{3. $Z$ is closed and $\langle\lambda, \check{\alpha}\rangle \in \mathbb{Z}_{\geq 0}$ for all $\alpha \in \Phi^{+}$.}

Following $\S 5$ of [Vogan 2], we now recall a few things about nilpotent cones. Via the Killing form, we identify $\mathfrak{g}$ with $\mathfrak{g}^{*}: \xi \in \mathfrak{g}$ is identified to the linear functional sending $\nu \in \mathfrak{g}$ to $\langle\xi, \nu\rangle$; we will use the same notation for the identified one. Let $\mathscr{N}$ be the nilpotent cone in $\mathfrak{g}$, and $\mathscr{N}^{*}$ the corresponding cone in $\mathfrak{g}^{*} . \mathscr{N}^{*}$ is also the variety defined by the ideal of homogeneous $G$ invariant polynomials of positive degree on $g^{*}(5.4,5.11$ in [Vogan 2]). Set

$$
\mathscr{N}_{\mathfrak{k}}^{*}=\left\{\xi \in \mathscr{N}^{*}:\left.\xi\right|_{\mathfrak{k}}=0\right\}=\mathscr{N} \cap(\mathfrak{g} / \mathfrak{k})^{*} .
$$

Note that $K$ acts on $\mathscr{N}_{\mathfrak{k}}^{*}$ via the coadjoint action with a finite number of orbits [Kostant-Rallis].

We shall use the same notations attached to the fixed data as in $\S 1$. Let $S$ be the set of all simple compact roots and $\langle S\rangle$ the set of all roots spanned by $S$. This choice then determines a parabolic subalgebra $\mathfrak{q}=\mathfrak{l}+\mathfrak{u}^{-}$:

$$
\mathfrak{l}=\mathfrak{h}+\sum_{\alpha \in\langle S\rangle} \mathfrak{g}^{\alpha}, \quad \mathfrak{u}^{-}=\sum_{\alpha \in \Phi^{+}-\langle S\rangle} \mathfrak{g}^{-\alpha} .
$$

Write $\pi$ for the natural map from $X$ to $X_{S}$, the flag variety of all parabolic subalgebras of type $S$. The orbit $Z$ is then mapped onto $Z_{1}$, the closed $K$ orbit containing the parabolic $q$. By the choice of $S$, we have $L \subset K$ and $\pi^{-1}\left(Z_{1}\right)=Z$. We use $\gamma_{1}$ to denote the moment map from $T^{*} X_{S}$ to $\mathfrak{g}^{*}$. Since $Z$ is a $K$-orbit, the conormal bundle $T_{Z}^{*} X$ is mapped into $\mathscr{N}_{\mathfrak{k}}^{*}$ under the moment map. Also $T_{Z}^{*} X$ is an irreducible variety and, $\gamma$ being $K$-equivariant, its image under $\gamma$ is the closure of a single $K$-orbit; call it $\mathscr{O}^{Z}$ :

2.4. Definition. $\gamma\left(T_{Z}^{*} X\right)$ is the closure of $\mathscr{O}^{Z}$ in $\mathscr{N}_{\mathfrak{k}}^{*}$.

Again by the choice of $S$, under $\gamma_{1}$, the conormal $T_{Z_{1}}^{*} X_{S}$ has the same image $\bar{\sigma}^{Z}$. We now state a condition which reflects the complexity of the moment map.

2.5. Assumption. The map $\gamma_{1}: T_{Z_{1}}^{*} X_{S} \rightarrow \overline{\mathscr{O}}^{Z}$ is generically finite.

Since $\gamma_{1}$ is $K$-equivariant, Assumption 2.5 is equivalent to that the above map, over the $K$-orbit $\mathscr{O}^{Z}$, is finite. We will discuss the validity of this condition later. For the following, note that the fiber of $\pi$ over the point $\mathfrak{q} \in X_{S}$ is given by $L / L \cap B$. 
2.6. Theorem. Suppose that the data $(Z, \tau, \lambda)$ satisfying 2.3 gives rise to a discrete series Harish-Chandra module I. If moreover Assumption 2.5 is satisfied with degree $d$, then

$$
\mathscr{V}(I)=d\left(\operatorname{dim} H^{0}\left(L / L \cap B, \text { ind } \tau \otimes \operatorname{det} \mathcal{N}_{Z \mid X}\right)\right) \cdot \overline{\mathscr{O}}^{Z} .
$$

Proof. Let $p$ be the projection $T^{*} X$ onto $X$, then $\operatorname{gr} \mathscr{D}_{\lambda}=p_{*} \mathscr{O}_{T^{*} X}$ with respect to the filtration by order of derivatives. Also, Lemma 1.2 implies

$$
\operatorname{gr} \mathscr{I}=i_{*}\left(\text { ind } \tau \otimes \operatorname{det} \mathscr{N}_{Z \mid X} \otimes \mathscr{N}_{Z \mid X}^{(\cdot)}\right) \text {. }
$$

Here $\mathscr{A}^{(\cdot)}$ is the whole symmetric algebra of $\mathscr{A}$. Thus as $\mathscr{O}_{T^{*} X^{-}}$-module,

$$
\operatorname{gr} \mathscr{I}=j_{*} q^{*}\left(\text { ind } \tau \otimes \operatorname{det} \mathscr{N}_{Z \mid X}\right),
$$

where $q$ is the projection of the conormal bundle $T_{Z}^{*} X$ onto $Z$ and $j$ is the closed imbedding of $T_{Z}^{*} X$ into $T^{*} X$. By Theorem 1.4, under our assumption, $\mathscr{V}(I)=\operatorname{Supp}\left(\gamma_{*} \operatorname{gr} I\right)$. Since $j$ is a closed imbedding, we shall restrict $\operatorname{gr} I$ to $T_{Z}^{*} X$ for the computation. Note that, $\gamma$ being proper and $K$-equivariant, for each $k$, the direct image sheaf $R^{k} \gamma_{*}$ gr $I$ is locally free on $\mathscr{O}_{Z}$ (by Grauert's theorem, see [Hartshone]). Thus $\mathscr{V}(I)$ is given by $\overline{\mathscr{O}}^{Z}$, the support of $\gamma_{*}(\operatorname{gr} I)$, with multiplicity $\left.\operatorname{dim} R^{0} \gamma_{*}(\operatorname{gr} I)\right|_{\xi}$; here $\xi \in \mathscr{O}^{Z}$ is a generic point in $\mathfrak{u}^{-} \cap \mathfrak{p}$, and $\left.\right|_{\xi}$ indicates the restriction to $\xi$. To compute the multiplicity, consider the following commutative diagram:

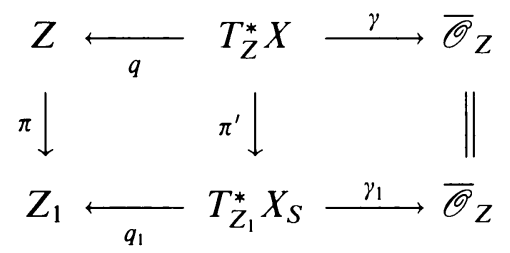

where $\pi^{\prime}$ is defined via the natural projection:

$$
T_{Z}^{*} X=K \times_{K \cap G}\left(\mathfrak{u}^{-} \cap \mathfrak{p}\right) \rightarrow K \times_{K \cap Q}\left(\mathfrak{u}^{-} \cap \mathfrak{p}\right)=T_{Z_{1}}^{*} X_{S} .
$$

Notice that the left square is a cartesian square, $\pi, \pi_{1}$ are proper, and $q^{*}, q^{* *}$ are exact. Denote by $x$ the point corresponding to $\mathfrak{b}$ in $X$ and $x_{1}$ its image in $X_{S}$. We have

$$
\begin{aligned}
\left.R^{k} \gamma_{*}(\operatorname{gr} I)\right|_{\xi} & =\left.R^{k} \gamma_{*} q^{*}\left(\text { ind } \tau \otimes \operatorname{det} \mathscr{N}_{Z \mid X}\right)\right|_{\xi} \\
& =\left.d \cdot R^{k} \pi_{*}^{\prime} q^{*}\left(\text { ind } \tau \otimes \operatorname{det} \mathscr{N}_{Z \mid X}\right)\right|_{\left(x_{1}, \xi\right)} \quad \text { by Assumption } 2.5 \\
& =\left.d \cdot q_{1}^{*} R^{k} \pi_{*}\left(\text { ind } \tau \otimes \operatorname{det} \mathscr{N}_{Z \mid X}\right)\right|_{\left(x_{1}, \xi\right)} \quad \text { by the base change } \\
& =\left.d \cdot R^{k} \pi_{*}\left(\text { ind } \tau \otimes \operatorname{det} \mathscr{N}_{Z \mid X}\right)\right|_{x_{1}} \\
& =d \cdot H^{k}\left(L / L \cap B, \operatorname{ind} \tau \otimes \operatorname{det} \mathscr{N}_{Z \mid X}\right) .
\end{aligned}
$$

In the above, $d \cdot *$ means $d$ copies of $*$. Setting $k=0$, we get the theorem.

2.8. Corollary. In the setting of Theorem $2.6, R^{k} \gamma_{*}(\operatorname{gr} I)=0$ on $\mathscr{O}^{Z}$ for $k>$ 0 .

Note that under the assumption, the character $d \tau+2 \rho_{n}$ on $L \cap B$ is $\mathbb{R}$ dominant (cf. the proof of Theorem 1.4); here $2 \rho_{n}=2\left(\rho-\rho_{\mathfrak{k}}\right)$ is the character 
of $\operatorname{det} \mathscr{N}_{Z \mid X}$. The corollary then follows from the proof of Theorem 2.6 and the Borel-Weil theorem.

Removing Assumption 2.5, we have the following formal result. Retaining the same notations in the proof of Theorem 2.6, the global section space $\Gamma(L / L \cap B$, ind $\tau)$ is then an $L$-module, hence extends trivially to a $K \cap Q$ module. It can then be induced to an invertible sheaf on $Z_{1}$.

2.9. Corollary. Suppose the data $(Z, \tau, \lambda)$ satisfying 2.3 gives rise to a discrete series Harish-Chandra I, then

$$
\mathscr{V}(I)=\operatorname{dim} H^{0}\left(\gamma_{1}^{-1}(\xi),\left.q_{1}^{*}\left(\operatorname{ind} \Gamma(L / L \cap B \text {,ind } \tau) \otimes \operatorname{det} \mathcal{N}_{Z_{1} \mid X_{S}}\right)\right|_{\gamma_{1}^{-1}(\xi)}\right) \cdot \overline{\mathscr{O}}^{Z} .
$$

This is a corollary of the proof of Theorem 2.6. Simply notice that $\gamma_{*} q^{*}$ $=\gamma_{1 *} \pi_{*}^{\prime} q^{*}=\gamma_{1 *} q_{1}^{*} \pi_{*}$, and $\pi_{*}\left(\right.$ ind $\left.\tau \otimes \operatorname{det} \mathcal{N}_{Z \mid X}\right)=$ ind $\Gamma(L / L \cap B$, ind $\tau) \otimes$ $\operatorname{det} \mathscr{N}_{Z_{1} \mid X_{S}}$.

Theorem 2.6 would compute the associated cycles of the discrete series explicitly if Assumption 2.5 is satisfied. Unfortunately this condition is generally not satisfied. The following is an example pointed out to us by D. Vogan. Consider $\mathrm{SU}(4,1)$ with the simple roots $\mathrm{c}-\mathrm{n}-\mathrm{n}-\mathrm{c}$ in its Dynkin diagram; c (resp. n) stands for compact (resp. noncompact) root. In this example, the conormal bundle $T_{Z_{1}}^{*} X_{S}$ has dimension 8 , but the orbit $\mathscr{O}^{Z}$ has dimension 7 . In a slightly different context, the complexity of the moment map is studied in [King]. We now consider a special case where Assumption 2.5 is satisfied. Recall from [Hecht-Schmid],

2.10. Lemma. In the above setting, suppose that $G_{\mathbb{R}}$ is simple. Then there exists a positive system $\Phi^{+}$in $\Phi$, for which exactly one simple root is noncompact, and such that the unique noncompact simple root occurs in the highest root with multiplicity one or two.

Such a positive system, in case $G_{\mathbb{R}}$ is simple, will be referred to as a Borelde Siebenthal (or simply B-S) system; the same terminology also applies to the $K$-orbit in $X$ determined by a such system.

2.11. Proposition. If the $K$-orbit $Z$ is Borel-de Siebenthal, then Assumption 2.5 is satisfied; in the multiplicity two case, $G_{\mathbb{R}}$ is assumed to be split.

Let $\Phi^{+}$be a B-S system, and as before $S$ is the set of all compact simple roots. Denote by $\Phi_{c}$ (resp. $\Phi_{n}$ ) for the set of compact (resp. noncompact) roots, and $\beta$ the unique simple noncompact root. Then $\Phi_{n}$ consists of roots in which $\beta$ occurs multiplicity 1 . Denote by $\Psi$ the set of roots in which $\beta$ occurs with multiplicity 2 . Thus $\Phi_{c}$ is the union of $\langle S\rangle$ and $\Psi$. Set

$$
\mathfrak{p}_{ \pm}=\bigoplus_{\alpha \in \Phi_{n}^{+}} \mathfrak{g}^{ \pm \alpha} ; \quad \mathfrak{q}_{ \pm}=\bigoplus_{\alpha \in \Psi^{+}} \mathfrak{g}^{ \pm \alpha}
$$

Note that $\mathfrak{p}_{ \pm}$are complex conjugates to each other; same for $\mathfrak{q}_{ \pm}$. Also $\mathfrak{p}=$ $\mathfrak{p}_{+} \oplus \mathfrak{p}_{-}$and $\mathfrak{u}_{-}=\mathfrak{p}_{-} \oplus \mathfrak{q}_{-}$.

\subsection{Lemma.}

(1) $\left[\mathfrak{l}, \mathfrak{p}_{ \pm}\right] \subset \mathfrak{p}_{ \pm},\left[\mathfrak{p}_{-}, \mathfrak{p}_{-}\right] \subset \mathfrak{q}_{-},\left[\mathfrak{p}_{-}, \mathfrak{q}_{-}\right]=\left[\mathfrak{q}_{-}, \mathfrak{q}_{-}\right]=0$.

(2) $\mathfrak{k}=\mathfrak{q}_{+} \oplus \mathfrak{l} \oplus \mathfrak{q}_{-}$, and $\mathfrak{l} \oplus \mathfrak{q}_{-}$is a maximal parabolic subalgebra of $\mathfrak{k}$.

(3) In case $\beta$ occurs with multiplicity 2 in the highest root, then for $\alpha_{1} \in$ $\Phi^{+}-\langle S\rangle$ and $\alpha_{2} \in \Psi^{+}$, we have $\left\langle\alpha_{1}, \alpha_{2}\right\rangle \geq 0$. 
This is just a simple observation (cf. Lemmas 4.11, 4.12 in [Hecht-Schmid]). For Proposition 2.11, since $\left(T_{Z_{1}}^{*} X_{S}\right)_{\mathfrak{q}}=\mathfrak{p}_{+}^{*} \simeq \mathfrak{p}_{-}$, it suffices to show that $K \cap Q$ has a dense open orbit in $\mathfrak{p}_{-}$and the isotropy subgroup in $K$ of an element in that dense orbit has its Lie algebra contained in $\mathfrak{k} \cap \mathfrak{q}$.

In case $\beta$ occurs with multiplicity 1 in the highest root, then $K=L$ and $\mathfrak{p}_{+}^{*} \subset \mathscr{N}_{\mathfrak{k}}$ is $\operatorname{Ad} K$-invariant, therefore the above two conditions are satisfied; in fact the degree in 2.5 is one. Moreover, $K_{\mathbb{R}}=G_{\mathbb{R}} \cap\left(K \cdot P_{ \pm}\right)$and the natural complex structure on $G /\left(K \cdot P_{+}\right)$then induces one on the symmetric space via the imbedding $G_{\mathbb{R}} / K_{\mathbb{R}} \hookrightarrow G /(K \cdot P)$. The totality of the standard modules $I$ arising from this situation constitutes the holomorphic discrete series. We summarize this case in the following corollary. Set $\mu=\lambda-\rho+2 \rho_{n}$, then $\Gamma\left(L / L \cap B\right.$, ind $\left.\tau \otimes \operatorname{det} \mathscr{N}_{Z \mid X}\right)$ is an irreducible $K$-module containing the highest weight $\mu$; call it $V_{\mu}$.

\subsection{Corollary. In the setting of Theorem 2.6, if I belongs to the holomorphic} discrete series, then $\mathscr{V}(I)=\left(\operatorname{dim} V_{\mu}\right) \cdot \mathfrak{p}_{+}^{*}$.

For the rest of this section, we assume that $\beta$ occurs with multiplicity 2 in the highest root. Let $x \in \mathfrak{q}_{-}$and $y \in \mathfrak{p}_{-}$be arbitrary; so $x+y$ is an arbitrary element in $\mathfrak{u}_{-}$. In view of Lemma $2.12(1), P_{-} \cdot y-y \subset \mathfrak{q}_{-}$, therefore the $\mathfrak{p}_{-}-$ components of $Q \cdot(x+y)=L P_{-} Q_{-} \cdot(x+y)$ are given by $L \cdot y$. Suppose now that $x+y$ is in the Richardson orbit corresponding to $Q$, then $L \cdot y$ is dense in $\mathfrak{p}_{-}$. In view of Lemma 2.12(1) and (2), the isotropy subgroup condition for $y$ amounts to the triviality of the centralizer $z_{\mathfrak{q}_{+}}(y)$ of $y$ in $\mathfrak{q}_{+}$, which is just the kernel of the map $f_{y}: \mathfrak{q}_{+} \rightarrow \mathfrak{p}_{+}$given by $z \mapsto[z, y]$ (note that $\left[\mathfrak{q}_{+}, y\right]$ is a subspace of $\mathfrak{p}_{+}$by Lemma 2.12(1)). Since the function $x \mapsto \operatorname{dim} f_{x}\left(\mathfrak{q}_{+}\right)$from $\mathfrak{p}_{-}$to $\mathbb{Z}$ is upper semicontinuous, the triviality of $z_{\mathfrak{q}_{+}}(y)$ follows from

2.14. Lemma. There exists $x \in \mathfrak{p}_{-}$with $z_{\mathfrak{q}_{+}}(x)=0$.

We recall that in [Vargas], a family of subroot systems $\Phi=\Phi^{0}, \Phi^{1}, \ldots, \Phi^{m}$ is defined inductively: $\Phi^{j+1}$ is the set of roots in $\Phi^{j}$ orthogonal to the noncompact simple roots for $\Phi^{j} \cap \Phi^{+}$, until the process stops. Set $S_{0}$ to be the set of all positive noncompact roots that are simple roots for some $\Phi^{i} \cap \Phi^{+}$, $0 \leq i \leq m$. Then $S_{0}$ is a strongly orthogonal set which spans $\Phi$ over $\mathbb{Q}$ (Lemma 2 of [Vargas]).

2.15. Lemma. For any $\alpha \in \Phi_{n}^{+}$, there is at most one $\gamma \in S_{0}$ such that $\alpha+\gamma$ is a root.

Proof. Suppose not, say $\gamma_{1} \neq \gamma_{2}$ both in $S_{0}$, and $\alpha+\gamma_{1}, \alpha+\gamma_{2}$ are both roots (hence in $\Psi^{+}$). By the orthogonality of $S_{0}$ and Lemma 2.12(3), $\left\langle\alpha, \gamma_{2}\right\rangle=$ $\left\langle\alpha+\gamma_{1}, \gamma_{2}\right\rangle \geq 0$; similarly $\left\langle\alpha, \gamma_{1}\right\rangle \geq 0$. Thus $\left\langle\alpha+\gamma_{1}, \alpha+\gamma_{2}\right\rangle \geq\langle\alpha, \alpha\rangle>0$. Therefore their difference which is given by $\gamma_{1}-\gamma_{2}$, is also a root. This then contradicts the strong orthogonality of $S_{0}$.

For Lemma 2.14, let $x$ be the sum of arbitrarily fixed nonzero root-vectors with roots ranging over $-S_{0}$. An arbitrary element $z \in \mathfrak{q}_{+}$is a linear combination of root-vectors in $\mathfrak{q}_{+}$. Let $\delta \in \Psi^{+}$be such that a root vector of which occurs nontrivially in $z \neq 0$. Since $S_{0}$ spans $\Phi^{+}$over $\mathbb{Q}$, there exists $\gamma \in S_{0}$ such that $\langle\delta, \gamma\rangle \neq 0$, which is then positive by 2.12(3). Therefore $\alpha:=\delta-\gamma$ is a root; which lies in $\Phi_{n}^{+}$by 2.12(1). Thus a nonzero root-vector for $\alpha$ occurs nontrivially in $[z, x]$ and by Lemma 2.15 , it cannot be cancelled by the 
contributions of other root-vectors occurring in $z$. It follows that $[z, x] \neq 0$, and the proof of Lemma 2.14 (and therefore that of 2.11) is completed.

3

In this section, we recall the basics of [Barbasch-Vogan] and Rossmann's Kirillov formula [Rossmann 1, 2]. Denote by $\boldsymbol{\theta}_{\lambda}$ the distribution character for the discrete series Harish-Chandra module $I_{\lambda}$ arising from a set of data satisfying the condition 2.3. As before, the subscript will often be omitted. Let $\mathfrak{g}_{\mathbb{R}}^{0}$ be an open $G_{\mathbb{R}}$-stable neighborhood of the origin in $\mathfrak{g}_{\mathbb{R}}$ such that the map exp: $\mathfrak{g}_{\mathbb{R}}^{0} \rightarrow G_{\mathbb{R}}$ is a diffeomorphism onto an open neighborhood of the identity in $G_{\mathbb{R}}$. Set $p(x)=\operatorname{det}^{1 / 2}(\sinh (\operatorname{ad} x / 2) /(\operatorname{ad} x / 2))$ on $\mathfrak{g}_{\mathbb{R}}^{0}$. For $\phi \in C_{0}^{\infty}\left(\mathfrak{g}_{\mathbb{R}}^{0}\right)$, we set $f_{\phi} \in C_{0}^{\infty}\left(\exp _{\mathfrak{g}_{\mathbb{R}}^{0}}^{0}\right)$ by $f_{\phi}(\exp x)=p(x)^{-1} \phi(x)$. Define a distribution on $\mathfrak{g}_{\mathbb{R}}^{0}$ by

$$
\left(\theta_{\lambda}, \phi\right)=\left(\boldsymbol{\Theta}_{\lambda}, f_{\phi}\right) .
$$

Now, for $t>0$, set $\phi_{t}(x)=t^{-\operatorname{dim} \mathfrak{g R}_{\mathrm{R}}} \phi\left(t^{-1} x\right)$.

3.2. Theorem (Barbasch-Vogan). There exist an integer $d$ and tempered distributions $D_{i},-d \leq i<\infty$, on $\mathfrak{g}_{\mathbb{R}}$ such that for $\phi \in C_{0}^{\infty}\left(\mathfrak{g}_{\mathbb{R}}\right)$,

$$
\left(\theta_{\lambda}, \phi_{t}\right) \sim \sum_{i=-d}^{\infty} t^{i}\left(D_{i}, \phi\right) \text { as } t \rightarrow 0^{+} .
$$

Furthermore the Fourier transform $\widehat{D}_{-d}$ of the leading term is a linear combination of invariant measures on $G_{\mathbb{R}}$-orbits of dimension $2 d$ in $\mathfrak{g}_{\mathbb{R}}^{*}$.

For $\xi \in \mathfrak{g}_{\mathbb{R}}^{*}$, we denote by $\Omega_{\xi}$ the coadjoint $G_{\mathbb{R}}$ orbit of $\xi$. The tangent space $T_{\xi} \Omega_{\xi}$ is naturally identified as $\mathfrak{g}_{\mathbb{R}} / \mathfrak{g}_{\mathbb{R}}(\xi)$, where

$$
\mathfrak{g}_{\mathbb{R}}(\xi)=\left\{x \in \mathfrak{g}_{\mathbb{R}}: \xi([x, y])=0 \forall y \in \mathfrak{g}_{\mathbb{R}}\right\} .
$$

The invariant fundamental symplectic 2-form $\sigma_{\xi}$ and Liouville measure $\beta_{\xi}$ are given by: at $\xi, X, Y \in T_{\xi} \Omega_{\xi}$,

$$
\sigma_{\xi}(\bar{X}, \bar{Y})=\xi([X, Y]) ; \quad \beta_{\xi}=\frac{\sigma_{\xi}^{m}}{(2 \pi)^{m} m !} .
$$

Here $2 m=\operatorname{dim} \Omega_{\xi}$. The following simple property of invariant measures under the dilation is useful.

3.4. Lemma. For $t>0$, let $L_{t}$ be the map $\xi \mapsto t \xi$. Then

$$
L_{t}^{*} \beta_{\Omega}=t^{m} \beta_{t \cdot \Omega} .
$$

This follows from (3.3) and the change of variables formula for integration. We now recall Rossmann's Kirillov formula: for $\phi \in C_{0}^{\infty}\left(\mathfrak{g}_{\mathbb{R}}^{0}\right)$,

$$
\left(\Theta_{\lambda}(\exp x), \phi(x)\right)=\int_{\Omega_{-i \lambda}} \int_{\mathfrak{g}_{\mathbf{R}}} e^{i(\xi, x)} \phi(x) p(x)^{-1} d x d \beta(\xi) .
$$

Note that $i \lambda \in \mathfrak{g}_{\mathbb{R}}^{*}$. Putting together (3.1) and (3.5), we have

$$
\left(\theta_{\lambda}, \phi\right)=\int_{\Omega_{-\downarrow \lambda}} \int_{\mathbf{g}_{\mathbf{R}}} e^{i(\xi, x)} \phi(x) d x d \beta\left(t^{-1} \xi\right) .
$$


Write $\hat{\phi}(\xi)=\int_{\mathfrak{g}_{\mathbf{R}}} e^{i(\xi, x)} \phi(x)$. Combining Lemma 3.4 and (3.6),

$$
\left(\theta_{\lambda}, \phi_{t}\right)=t^{-m}\left(\beta_{-i t \lambda}, \hat{\phi}\right) .
$$

Therefore the asymptotics of $\theta_{\lambda}$ is the same as that of the Fourier transform of $\beta_{-i t \lambda}$ as $t \rightarrow 0^{+}$.

We remark that the asymptotic expansion of [Barbasch-Vogan] holds for any irreducible Harish-Chandra module, and the Kirillov formula holds for any irreducible tempered representation [Rossmann 1, 2]. We conclude this section with the following special case.

3.8. Theorem. Suppose $G_{\mathbb{R}}$ is compact and $V_{\mu}$ an irreducible $G_{\mathbb{R}}$-module containing $\mu$ as a highest weight (with respect to a choice of positive system). Then

$$
t^{-m} \beta_{-i t(\mu+\rho)} \rightarrow\left(\operatorname{dim} V_{\mu}\right) \delta_{0} \text { as } t \rightarrow 0^{+} .
$$

First of all, since $G_{\mathbb{R}}$ is not connected in general, an irreducible module is not determined by its highest weights; nevertheless its dimension is determined by the highest weights (see $\S 5.1$ of [Vogan 1]). The theorem then follows from the Kirillov formula

$$
\operatorname{dim} V_{\mu}=\int_{\Omega_{-i(\mu+\rho)}} d \beta=\operatorname{vol}\left(\Omega_{-i t(\mu+\rho)}\right),
$$

and the fact that $\Omega_{-i t(\mu+\rho)} \rightarrow\{0\}$ as $t \rightarrow 0^{+}$.

\section{4}

In this section, we derive an integration formula for invariant measures in the context of a fibration. As in 2.1 , we assume that $G_{\mathbb{R}}$ contains a compact Cartan subgroup $H_{\mathbb{R}}$. Retaining notations of $\S 2$, so $S$ is the subset of simple compact roots in $\Phi^{+}$. Denote the center of $\mathfrak{l}$ by $\mathfrak{h}_{S}$, which is contained in $\mathfrak{h}$; and by $\mathfrak{h}^{S}$ its orthogonal complement w.r.t. the invariant Killing form. These are all defined over $\mathbb{R}$. Let $\chi$ be a fixed regular element in $\mathfrak{h}_{\mathbb{R}}^{*}$. Write

$$
\chi=\chi_{1}+\chi_{2}, \quad \text { with } \chi_{1} \in\left(\mathfrak{h}^{S}\right)^{*}, \quad \chi_{2} \in\left(\mathfrak{h}_{S}\right)^{*} .
$$

Set, for $s, t>0,(s, t) \chi=s \chi_{1}+t \chi_{2}$. The parabolic $\mathfrak{q}$ is the $\theta$-stable parabolic subalgebra defined by $\chi_{2}$ in the sense of [Vogan 2]. Denote by $L_{\mathbb{R}}$ the real point of the Levi part and $L_{\mathbb{R}}^{\prime}$ for its derived group. In our setting of $G_{\mathbb{R}}$ (cf. $\S 1$ ) and the choice of $S, L_{\mathbb{R}}$ is the centralizer of $\chi_{2}$ in $G_{\mathbb{R}}$. Note that the Killing form identifies $\mathfrak{h}_{\mathbb{R}}^{*}$ to a subspace in $\mathfrak{g}_{\mathbb{R}}^{*}$.

4.2. Lemma. There is a $G_{\mathbb{R}}$-equivariant fibration $\pi: \Omega_{(s, t) \chi} \rightarrow \Omega_{t \chi_{2}}$ with fibers isomorphic to $L_{\mathbb{R}} / H_{\mathbb{R}} \simeq L_{\mathbb{R}}^{\prime} /\left(H_{\mathbb{R}} \cap L_{\mathbb{R}}^{\prime}\right)$.

Proof. The map is given by $g \cdot(s, t) \chi \mapsto g \cdot t \chi_{2}$ for $g \in G_{\mathbb{R}}$. This is well defined since the isotropy subgroup at $t \cdot \chi_{2}$ is $L_{\mathbb{R}}$, and that at $(s, t) \cdot \chi$ is $H_{\mathbb{R}}$.

In the following, we use the superscript $L$ to indicate objects defined with respect to $L_{\mathbb{R}}$. Set $2 m=\operatorname{dim} \Omega_{(s, t) \chi}, 2 p=\operatorname{dim} \Omega_{t \chi_{2}}$, and $2 q=\operatorname{dim} \Omega_{s \chi_{1}}^{L}$; then $m=p+q$. Define an invariant (degenerate) 2-form on $\Omega_{(s, t) \chi}$ by: at $(s, t) \chi, \bar{X}, \bar{Y} \in \mathfrak{g}_{\mathbb{R}} / \mathfrak{h}_{\mathbb{R}}$,

$$
\sigma_{s \chi_{1}}^{\prime}(\bar{X}, \bar{Y}):=s \chi_{1}([X, Y]) .
$$


This is well defined since $\mathfrak{h}_{\mathbb{R}} \subset \mathfrak{g}_{\mathbb{R}}\left(s \chi_{1}\right)$. At points $(s, t) \chi$ and $t \chi_{2}$, the tangent map $\pi_{*}$ of the above fibration is just the projection in the short exact sequence

$$
0 \rightarrow \mathfrak{l}_{\mathbb{R}} / \mathfrak{h}_{\mathbb{R}} \rightarrow \mathfrak{g}_{\mathbb{R}} / \mathfrak{h}_{\mathbb{R}} \rightarrow \mathfrak{g}_{\mathbb{R}} / \mathfrak{l}_{\mathbb{R}} \rightarrow 0,
$$

which admits an ( $L_{\mathbb{R}}$-equivariant) splitting by the orthogonal projection from $\mathfrak{g}_{\mathbb{R}}$ onto $\mathfrak{l}_{\mathbb{R}}$. Thus we can think of the 2 -form $\sigma_{s \chi_{1}}^{L}$ as an invariant 2-form defined on $\Omega_{(s, t) \chi}$. In other words, this is the 2 -form given by $\left(g^{-1}\right)^{*} \sigma_{s \chi_{1}}^{L}$ on the fiber $\pi^{1}\left(g \cdot \chi_{2}\right)$. It is clear that $\sigma_{(s, t) \chi}=\pi^{*} \sigma_{t \chi_{2}}+\sigma_{s \chi_{1}}^{\prime}$. Therefore, writing in terms of the fibration, we have

$$
\sigma_{(s, t) \chi}=\sigma_{s \chi_{1}}^{L}+\left(\sigma_{s \chi_{1}}^{\prime}-\sigma_{s \chi_{1}}^{L}+\pi^{*} \sigma_{t \chi_{2}}\right)
$$

where $\sigma_{(s, t) \chi}, \sigma_{s \chi_{1}}^{L}$, and $\sigma_{s \chi_{1}}^{\prime}-\sigma_{s \chi_{1}}^{L}+\pi^{*} \sigma_{t \chi_{2}}$ have ranks $m, q$, and $p$ respectively. Taking the $m$ th exterior power we have

\subsection{Proposition.}

$$
\beta_{(s, t) \chi}=\beta_{s \chi_{1}}^{L} \cdot\left(\pi^{*} \beta_{t \chi_{2}}+\Psi(s)\right),
$$

where $\Psi(s)$ is a $2 p$-form depending analytically on $s$ and $\Psi(0)=0$.

Here again $\beta_{s \chi_{1}}^{L}$ is understood as the $2 q$-form given by $\left(g^{-1}\right)^{*} \beta_{s \chi_{1}}^{L}$ on each fiber over $g \cdot t \chi_{2}$. The property of $\Psi$ follows from the fact that, at $s=0$, $\sigma_{s \chi_{1}}^{\prime}=\sigma_{s \chi_{1}}^{L}$ and $\pi^{*} \sigma_{t \chi_{2}}$ has rank $p$.

We now go back to the setting of $\S 2$ and begin the computation of the leading term of $\theta_{\lambda}$, the character of a discrete series representation. We use $-i \lambda$ for $\chi$ above. Now $\mathfrak{l} \subset \mathfrak{k}, L \subset K$, and the fiber of $\pi$ is compact and is given by $L / L \cap B$ in terms of the notations in $\S 2$. Also the condition 2.3 on $\lambda$ (i.e., being regular $\mathbb{R}$-dominant) implies that $\lambda_{1}$ is $\mathbb{R}$-dominant w.r.t. $\Phi^{+}\left(\mathfrak{l}^{\prime}, \mathfrak{h}^{S}\right)$. Recalling the notations in Theorem 2.6.

\subsection{Proposition. In the above setting, we have}

$$
t^{-q} \beta_{i t \lambda_{1}}^{L} \rightarrow\left(\operatorname{dim} H^{0}\left(L / L \cap B \text {, ind } \tau \otimes \operatorname{det} \mathscr{N}_{Z \mid X}\right)\right) \delta_{0} \quad \text { as } t \rightarrow 0^{+} .
$$

Proof. In view of Theorem 3.8, as $t \rightarrow 0^{+}$, the weighted orbital measure $t^{-q} \beta_{-i t \lambda_{1}}^{L}$ converges to $\delta_{0}$ with multiplicity given by the dimension of an irreducible $L_{\mathbb{R}}^{\prime}$-module containing the highest weight $\lambda-\rho\left(\Phi^{+}\left(\mathfrak{l}^{\prime}, \mathfrak{h}^{S}\right)\right)$ restricted to $\mathfrak{h}^{S}$. On the other hand, the multiplicity occurring in this proposition is given by that corresponding to the weight $\lambda-\rho+2 \rho_{n}$ for $\operatorname{det} \mathscr{N}_{Z \mid X}$ is induced by the weight $2 \rho_{n}$ (the sum of all noncompact positive roots; cf. Corollary 2.8). Now $\rho=\rho\left(\Phi^{+}\left(\mathfrak{l}^{\prime}, \mathfrak{h}^{S}\right)\right)+\rho(\mathfrak{u})$; it therefore suffices to check that $\rho(\mathfrak{u})$ and $\rho_{n}$ are both trivial on $\mathfrak{h}^{S}$. Since a basis of $\mathfrak{h}$ is given by fundamental dual weights of simple roots, we only need to show that for any $\alpha$ in $S,\langle\rho(\mathfrak{u}), \alpha\rangle=\left\langle\rho_{n}, \alpha\right\rangle=0$. The first equality holds for $\mathfrak{u}^{-}$is the nilradical of $\mathfrak{q}$. Since the simple reflection about $\alpha$ leaves the set of positive noncompact roots unchanged, the second equality follows.

In view of Proposition 4.4, to compute the limit of $\beta_{-i t \lambda}$, it remains to determine that of $\beta_{-i t \lambda_{2}}$.

To describe the limit of the invariant measure $\beta_{-i t \lambda_{2}}$, we need the following correspondence of nilpotent orbits. For details, for [Sekiguchi], [Vogan 2]. 
5.1. Theorem (Sekiguchi). There is a natural bijection between $G_{\mathbb{R}^{-} \text {orbits on }}$ $\mathscr{N}_{\mathbb{R}}^{*}$ and $K$-orbits on $\mathscr{N}_{\mathbb{R}}^{*}$. Suppose $\mathscr{O}_{\mathbb{R}}$ and $\mathscr{O}_{K}$ are a pair of corresponding $G_{\mathbb{R}}, K$-orbits respectively, then $\operatorname{dim}_{\mathbb{R}} \mathscr{O}_{\mathbb{R}}=2 \operatorname{dim}_{\mathbb{C}} \mathscr{O}_{K}$.

Here $\mathscr{N}_{\mathbb{R}}^{*}$ is $\mathscr{N} \cap \mathfrak{g}_{\mathbb{R}}^{*}$. Recall from $\S 2$ that $\mathscr{O}^{Z}$ is a $K$-orbit in $\mathscr{N}_{\mathfrak{q}}^{*}$. We set 5.2. Definition-Notation. $\mathscr{O}_{\mathbb{R}}^{Z}:=$ the $G_{\mathbb{R}}$-orbit on $\mathscr{N}_{\mathbb{R}}^{*}$ corresponding to $\mathscr{O}^{Z}$. Therefore $\operatorname{dim}_{\mathbb{R}} \mathscr{O}_{\mathbb{R}}^{Z}=2 \operatorname{dim}_{\mathbb{C}} \mathscr{O}^{Z}=2 \operatorname{dim}_{\mathbb{C}} X_{S}=2\left|\Phi^{+}-\langle S\rangle\right|$; set it to be $2 l$. To write down the correspondence explicitly, we need to work in $\mathfrak{g}$ (cf. [Sekiguchi] and [Vogan 2]). Identifying $\mathfrak{g}$ with $\mathfrak{g}^{*}$ as before, there exists $h \in \mathfrak{k}, e, f \in \mathfrak{p}$ such that

$$
\begin{array}{r}
K \cdot e=\mathscr{O}^{Z}, \quad[h, e]=2 e, \quad[h, f]=2 f, \quad[e, f]=h, e+f, \\
i(e-f) \in \mathfrak{p}_{\mathbb{R}}, \quad i h \in \mathfrak{k}_{\mathbb{R}} .
\end{array}
$$

In particular, $f=\bar{e}$. From this, we set

$$
h^{d}=i(e-f), \quad e^{d}=\frac{1}{2}(e+f+i h), \quad f^{d}=\frac{1}{2}(e+f-i h) ;
$$

then $\mathscr{O}_{\mathbb{R}}^{Z}=G_{\mathbb{R}} \cdot e^{d}$. Recalling notations in $\S 1$, the main result of this section is

5.5. Theorem. Suppose that the data $(Z, \tau, \lambda)$ gives rise to a holomorphic discrete series Harish-Chandra module $I_{\lambda}$. Then the leading term of the asymptotics of $\theta_{\lambda}$ is given by

$$
\widehat{D}_{-l}=\left(\operatorname{dim} V_{\mu}\right) \cdot \beta_{\mathscr{O}_{\mathbf{R}}^{Z}} .
$$

This together with Corollary 2.13 , then verifies a conjecture of D. Vogan in the special case of holomorphic discrete series representations.

In view of Theorem 3.8, and Propositions 4.4 and 4.5, Theorem 5.5 follows from

5.6. Proposition. Under the assumption of Theorem 5.5, $\beta_{-i t \lambda_{2}} \rightarrow \beta_{e^{d}}$ as $t \rightarrow$ $0^{+}$.

The proof of this consists of a few lemmas. Note that it suffices to prove this for $G_{\mathbb{R}}$ simple, which we assume for the rest of the proof. Under the assumption of Theorem $5.5, \mathfrak{k}=\mathfrak{l}$ has a one-dimensional center $\mathfrak{h}_{S}$ which we will denote by $\mathfrak{c}$ in the following. In particular, the connected component $K_{\mathbb{R}}^{0}$ of $K_{\mathbb{R}}$ acts trivially on $\mathfrak{c}$. Since the correspondence of orbits in Theorem 5.1 is most conveniently stated in the context of $\mathscr{N}$ (cf. (5.3), (5.4)), we shall work in that setting as well in the rest of this section. In particular, $i \lambda_{2}$ will be considered in $\mathfrak{g}_{\mathbb{R}}$.

5.7. Lemma. Let $\nu$ be a nilpotent element in $\mathfrak{g}_{\mathbb{R}}$ and $\chi \in \mathfrak{g}_{\mathbb{R}}$. Assume that $\Omega_{\nu}$ is a limit orbit of $\Omega_{t \chi}$ as $t \rightarrow 0^{+}$and $\operatorname{dim} \Omega_{\nu}=\operatorname{dim} \Omega_{t \chi}$ for $t>0$; then $\beta_{t x} \rightarrow \beta_{\nu}$ in a small neighborhood of $\Omega_{\nu}$ as $t \rightarrow 0^{+}$.

Proof. It suffices to show this in a neighborhood of $\nu$. It is standard (see [Varadarajan]) that transversal slices to $\Omega_{\nu}$ at $\nu$ exist: there are subspaces $S, U$ in $\mathfrak{g}_{\mathbb{R}}$ and the map

$$
\psi: S \times U \rightarrow \mathfrak{g}_{\mathbb{R}}, \quad(\xi, x) \mapsto e^{x} \cdot(\nu+\xi) \cdot e^{-x},
$$

is a diffeomorphism in a neighborhood, say $A \times B$ of $(0,0)$, onto a neighborhood $W$ of $\nu$ in $\mathfrak{g}_{\mathbb{R}}$ such that $p^{-1}(0)$ is mapped onto $\Omega_{\nu}$ locally in these neighborhoods ( $p$ is the projection onto $S$ ). In the following, we only consider 
the restriction of $\psi$ on the neighborhood $A \times B$. Since $\Omega_{\nu}$ is a limit orbit of $\Omega_{t \chi}$ as $t \rightarrow 0^{+}$, there exists $\xi_{t} \in S$ such that $\nu+\xi_{t} \in \Omega_{t \chi}$ if $0 \leq t \leq t_{0}$ for some $t_{0}>0$. Therefore for $0 \leq t \leq t_{0}, \psi\left(p^{-1}\left(\xi_{t}\right)\right) \subset \Omega_{t \chi}$, and the assumption on dimensions gives $\psi\left(p^{-1}\left(\xi_{t}\right)\right)=\Omega_{t \chi}$. Now as 2-forms on $\mathfrak{g}_{\mathbb{R}}, \sigma_{\nu+\xi_{t}} \rightarrow \sigma_{\nu}$. Also the projection of $U$ to $T_{\nu+\xi_{t}} \Omega_{t \chi}$ is an isomorphism if $0 \leq t \leq t_{0}$ for $\psi$ is a diffeomorphism. Thus we have $\sigma_{t \chi} \rightarrow \sigma_{\nu}$ and therefore $\beta_{t \chi} \rightarrow \beta_{\nu}$ in the neighborhood $W$ as $t \rightarrow 0^{+}$.

5.8. Lemma. (1) $\operatorname{dim}_{\mathbb{R}} \Omega_{-i t \lambda_{2}}=2 l$.

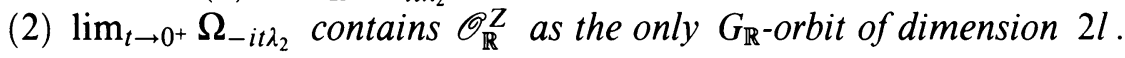

The first statement follows from $\Omega_{-i t \lambda_{2}} \simeq G_{\mathbb{R}} / K_{\mathbb{R}}$ and $l=\left|\Phi_{n}^{+}\right|$. For the proof of (2), denote by $\beta$ the unique noncompact simple root. Recall that $e, f, h$ and others are those in (5.3) and (5.4) specifying the orbit $\mathscr{O}^{Z}$.

5.9. Claim. $\mathscr{O}_{\mathbb{R}}^{Z}=G_{\mathbb{R}} \cdot e^{d} \subset \lim _{t \rightarrow 0^{+}} \Omega_{-i t \lambda_{2}}$.

Proof. Clearly we have $\exp \left(t h^{d}\right) \in G_{\mathbb{R}}$ and

$$
\lim _{t \rightarrow+\infty} e^{-2 t} \exp \left(t h^{d}\right) \cdot\left(e^{d}-f^{d}\right)=e^{d} .
$$

Recall from $\S 4$ that $i \lambda_{2}$ is supported on $\mathfrak{c}_{\mathbb{R}}$, the center of $\mathfrak{I}_{\mathbb{R}}$. Now the complex structure of $G_{\mathbb{R}} / K_{\mathbb{R}}$ is determined by ad $\mathfrak{c}_{\mathbb{R}}$ with eigenspaces $\mathfrak{p}_{ \pm}$(see [Helgason]). Since $e \in \mathfrak{p}_{-}$and $f=\bar{e}$, we have $\left[\lambda_{2}, e\right]=-a e,\left[\lambda_{2}, f\right]=-a f$ for some positive constant $a$. Also $\left\langle h, \lambda_{2}\right\rangle=\left\langle[e, f], \lambda_{2}\right\rangle=-a\langle e, f\rangle\langle 0$, thus $h=c \lambda_{2}+y$ for some negative constant $c$ and $y \equiv 0$ on $\mathfrak{c}_{\mathbb{R}}$. Now $h^{d}=i(e-f)$, a direct computation gives $\operatorname{ad} h^{d}\left(\lambda_{2}\right)=\frac{-a}{2}$ ad $h^{d}(h)$. Thus

$$
\exp \left(t h^{d}\right) \cdot h-h=\frac{-a}{2}\left(\exp \left(t h^{d}\right) \cdot \lambda_{2}-\lambda_{2}\right) .
$$

Therefore $\exp \left(t h^{d}\right) \cdot\left(e^{d}-f^{d}\right)=i \exp \left(t h^{d}\right) \cdot h=\frac{2}{a} \exp \left(t h^{d}\right) \cdot\left(-i \lambda_{2}\right)+i\left(h-\frac{2}{a} \lambda_{2}\right)$. Together with (5.10) this implies

$$
\lim _{t \rightarrow+\infty} e^{-2 t} \exp \left(t h^{d}\right) \cdot\left(-i \lambda_{2}\right)=\frac{a}{2} e^{d} .
$$

Thus $G_{\mathbb{R}} \cdot e^{d}=\mathscr{O}_{\mathbb{R}}^{Z} \subset \lim _{t \rightarrow 0^{+}} \Omega_{i t \lambda_{2}}$.

To finish the proof of Lemma 5.8 , since $\operatorname{dim} \mathscr{O}_{\mathbb{R}}^{Z}=2 l$, it remains to show that it is the only limit $G_{\mathbb{R}}$-orbit of dimension $2 l$. In view of our assumption on the group $G_{\mathbb{R}}$ (cf. $\S 1$ ), it suffices to show this for the connected component $G_{\mathbb{R}}^{0}$ of $G_{\mathbb{R}}$. In other words, $e^{d}$ as in Claim $5.9, G_{\mathbb{R}}^{0} \cdot e^{d}$ is the only $2 l$-dimensional limit orbit of $G_{\mathbb{R}}^{0} \cdot\left(-i t \lambda_{2}\right)$ as $t \rightarrow 0^{+}$. For the remainder of the proof, to avoid an extra load of notations, we assume that $G_{\mathbb{R}}$ is connected. Consider the function

$$
\phi: G_{\mathbb{R}} \rightarrow \mathbb{R} \quad \phi(g)=\left\langle g \cdot\left(-i \lambda_{2}\right),-i \lambda_{2}\right\rangle=-\left\langle g \cdot \lambda_{2}, \lambda_{2}\right\rangle .
$$

5.12. Claim. $\phi$ is continuous and $\phi(g)<0$ for all $g \in G_{\mathbb{R}}$.

Proof. In view of the Cartan decomposition $G_{\mathbb{R}}=K_{\mathbb{R}} A_{\mathbb{R}} K_{\mathbb{R}}$ where $A_{\mathbb{R}}$ is a maximal abelian subgroup in $\exp \mathfrak{p}_{\mathbb{R}}$, and $\lambda_{2} \in \mathfrak{h}_{S}^{*}$ on which $K_{\mathbb{R}}$ acts trivially, it suffices to check that

$$
\left\langle g \cdot \lambda_{2}, \lambda_{2}\right\rangle>0 \text { for all } g \in A_{\mathbb{R}} .
$$


Let $\gamma$ be a positive noncompact root, we can choose root vectors $X_{ \pm \gamma}$ such that $H_{\gamma}, X_{\gamma}, X_{-\gamma}$ satisfies the same condition 5.3 replacing $h, e, f$ respectively. Then for $c \in \mathbb{R}$, we have $Y_{\gamma}=c\left(X_{\gamma}+X_{-\gamma}\right) \in \mathfrak{p}_{\mathbb{R}}$. A direct computation gives, setting $g=\exp Y_{\gamma}$,

$$
g \cdot \lambda_{2}=\lambda_{2}+\frac{1}{2}\left\langle\gamma, \lambda_{2}\right\rangle\left[(\cosh (2 c)-1) H_{\gamma}+\sinh (2 c)\left(X_{\gamma}-X_{-\gamma}\right)\right] .
$$

Therefore

$$
\left\langle g \cdot \lambda_{2}, \lambda_{2}\right\rangle=\left\langle\lambda_{2}, \lambda_{2}\right\rangle+\frac{1}{2} \frac{\left\langle\gamma, \lambda_{2}\right\rangle^{2}}{\langle\gamma, \gamma\rangle}[\cosh (2 c)-1]>0 .
$$

An arbitrary element $Y \in \mathfrak{a}_{\mathbb{R}}$ is a linear combination of $Y_{\gamma}$ 's with $\gamma$ ranging in a maximal strongly orthogonal set in $\Phi_{n}^{+}$. Thus $\left\langle g \cdot \lambda_{2}, \lambda_{2}\right\rangle$ is a sum of positive numbers.

Suppose now that $\Omega$ is a $G_{\mathbb{R}}$-orbit of dimension $2 l$, different from $\mathscr{O}_{\mathbb{R}}^{Z}$, contained in the limit of $\Omega_{-i t \lambda_{2}}$ as $t \rightarrow 0^{+}$. Again by the correspondence of orbits in Theorem 5.1, we may assume that $\Omega=G_{\mathbb{R}} \cdot e^{d}$, for some $e^{d} \in \mathscr{N}_{\mathbb{R}}$, and $\left\{h^{d}, e^{d}, f^{d}\right\},\{h, e, f\}$ as given in (5.4) and (5.3). By Claim 5.12, it is enough to show that, for some $k \in K_{\mathbb{R}}, g \in A_{\mathbb{R}}$,

$$
\left\langle g k \cdot e^{d},-i \lambda_{2}\right\rangle>0 .
$$

Since ih $\in \mathfrak{k}_{\mathbb{R}}$, with conjugation by elements in $K_{\mathbb{R}}$ if necessary, we may assume that $i h \in \mathfrak{h}_{\mathbb{R}}$. Recall that $e^{d}=\frac{1}{2}(e+f+i h)$ and $e+f \in \mathfrak{p}_{\mathbb{R}}$. With $g$ as in (5.13), $\gamma \in \Phi_{n}^{+}$, we have

$$
\begin{aligned}
\left\langle 2 i e^{d}, g \cdot \lambda_{2}\right\rangle=-\left\langle h, \lambda_{2}\right\rangle+\frac{\left\langle\gamma, \lambda_{2}\right\rangle}{2}[ & -(\cosh 2 c-1)\left\langle H_{\gamma}, h\right\rangle \\
& \left.+(\sinh 2 c)\left\langle i(e+f), X_{\gamma}-X_{-\gamma}\right\rangle\right] .
\end{aligned}
$$

Therefore, if $\left\langle H_{\gamma}, h\right\rangle>0$ for some $\gamma$, we may choose $c \ll 0$ (respectively $0 \ll c)$ if $\left\langle i(e+f), X_{\gamma}-X_{-\gamma}\right\rangle \geq 0$ (respectively $\leq 0$ ) such that $\left\langle 2 i e^{d}, g \cdot \lambda_{2}\right\rangle<0$ and we are done. So assume that $\left\langle H_{\gamma}, h\right\rangle \leq 0$, for all $\gamma \in \Phi_{n}^{+}$. Now $\left\{X_{\gamma}: \gamma \in\right.$ $\left.\Phi_{n}\right\}$ is a basis of $\mathfrak{p}$, the negativity assumption on $h$ above and the condition $[h, e]=2 e$ implies that $e$ must be in $\mathfrak{p}_{-}$, the span of root-vectors of negative noncompact roots. In terms of notations in $\S 2, \mathfrak{p}_{-}=\mathfrak{u}_{-}$and $L=K$ under the Hermitian structure assumption, the dimension assumption on $\Omega$ then implies $K \cdot e=\mathscr{O}^{Z}$. Thus by Theorem $5.1, \Omega=\mathscr{O}_{\mathbb{R}}^{Z}$, which is a contradiction. This completes the proof of Lemma 5.8.

Proof of Proposition 5.6. First of all, by identifying $\beta_{-i t \lambda_{0}}$ with the invariant integral on $G_{\mathbb{R}} / K_{\mathbb{R}}$, it is standard that the limit exists (see [Rossman 2], [Varadarajan]). Lemmas 5.7 and 5.8 then imply that the limit is supported on the closure of $\mathscr{O}_{\mathbb{R}}^{Z}$ and on which it equals $\beta_{e^{d}}$. The homogeneity property in Lemma 3.4 then excludes those supported on the boundary.

\section{REFERENCES}

[Barbasch-Vogan] D. Barbasch and D. Vogan, The local structure of characters, J. Funct. Anal. 37 (1980), 27-55.

[Beilinson-Bernstein] A. Beilinson and J. Bernstein, Localisation de g-modules, C. R. Acad. Sci. Paris Ser. I 292 (1981), 15-18. 
[Borho-Brylinski] W. Borho and J.-L. Brylinski, Differential operators on homogeneous spaces. III, Invent. Math. 80 (1985), 1-68.

[Chang] J.-T. Chang, Special K-types, tempered characters and the Belinson-Bernstein realization, Duke Math. J. 56 (1988), 345-383.

[Griffiths] P. A. Griffiths, Hermitian differential geometry, Chern classes, and positive vector bundles, Global Analysis, Univ. of Tokyo Press, Tokyo, 1969, pp. 185-251.

[Hartshone] R. Hartshone, Algebraic geometry, Springer-Verlag, New York, 1979.

[Harish-Chandra] Harish-Chandra, Discrete series for semisimple Lie groups. II, Acta Math. 116 (1966), 1-111.

[Hecht-Schmid] H. Hecht and W. Schmid, A proof of Blattner's conjecture, Invent. Math. 31 (1975), 129-154.

[Helgason] S. Helgason, Differential geometry, Lie groups and symmetric spaces, Academic Press, New York, 1978.

[King] D. King, Character polynomials of discrete series representations, Lecture Notes in Math., vol. 880, Springer-Verlag, 1981, pp. 267-302.

[Kostant-Rallis] B. Kostant and S. Rallis, Orbits and representations associated with symmetric spaces, Amer. J. Math. 93 (1971), 753-809.

[Matsuki] T. Matsuki, The orbits of affine symmetric spaces under the action of minimal parabolic subgroups, J. Math. Soc. Japan 31 (1979), 332-357.

[Richardson] R. W. Richardson, Conjugacy classes in parabolic subgroups of semisimple algebraic groups, Bull. London Math. Soc. 6 (1974), 21-24.

[Rossmann 1] W. Rossman, Kirillov's character formula for reductive Lie groups, Invent. Math. 48 (1978), 207-220.

[Rossmann 2] W. Rossman, Limit characters of reductive Lie groups, Invent. Math. 61 (1980), 53-66.

[Rossmann 3] W. Rossman, Limit orbits in reductive Lie algebras, Duke Math. J. 49 (1982), 215229.

[Schmid 1] W. Schmid, Homogeneous complex manifolds and representations of semisimple Lie groups, Ph.D. Dissertation, Univ. of California at Berkeley, 1967.

[Schmid 2] __, On the characters of the discrete series (the Hermitian symmetric case), Invent. Math. 30 (1975), 47-144.

[Sekiguchi] J. Sekiguchi, Remarks on nilpotent orbits of a symmetric pair, J. Math. Soc. Japan 39 (1987), 127-138.

[Varadarajan] V. S. Varadarajan, Harmonic analysis on real reductive groups, Lecture Notes in Math., vol. 576, Springer-Verlag, New York, 1977.

[Vargas] J. A. Vargas, A character formula for the discrete series of a semi-simple Lie group, Bull. Amer. Math. Soc. (N.S.) 2 (1980), 465-467.

[Vogan 1] D. Vogan, Jr., Representations of real reductive Lie groups, Birkhäuser, Boston, Mass., 1981.

[Vogan 2] _ _ Associated varieties and unipotent representations, Preprint, 1990.

Department of Mathematics, Oklahoma State University, Stillwater, Oklahoma 74078-0613

E-mail address: changj@hardy.math.okstate.edu 